\title{
Non-Hopf Hypersurfaces in 2-dimensional Complex Space Forms
}

\author{
Mayuko KON
}

Shinshu University

(Communicated by K. Ahara)

\begin{abstract}
In this paper we give a geometric characterization of non-Hopf hypersurfaces in the complex space form $M^{2}(c)$ under a condition on the shape operator. We also classify pseudo-parallel real hypersurfaces of $M^{2}(c)$.
\end{abstract}

\section{Introduction}

It is an interesting problem to study real hypersurfaces immersed in the complex space form $M^{n}(c)$ under a condition on curvature tensor, or the Ricci tensor, or sectional curvature. In this paper we consider the case of the 2-dimensional complex space form $M^{2}(c)$. In [3], Ivey and Ryan constructed some examples of non-Hopf real hypersurfaces in the non-flat complex space form $M^{2}(c)$. Let $M$ be a real hypersurface in the complex hyperbolic space $\mathbf{C} H^{2}$ or the complex projective space $\mathbf{C} P^{2}$. We denote by $(\phi, \xi, \eta, g)$ an almost contact metric structure. At each $p \in M$, we define a subspace $\mathcal{H}_{p} \subset T_{p} M$ as the smallest subspace that contains the structure vector field $\xi$ and that is invariant under the shape operator $A$. We assume that $\mathcal{H}=\sqcup_{p} \mathcal{H}_{p}$ is a smooth two-dimensional distribution on $M$. Then we obtain an adapted orthonormal frame $\{\xi, X, \phi X\}$ with respect to which the shape operator has the form

$$
A=\left(\begin{array}{lll}
\alpha & h & 0 \\
h & \lambda & 0 \\
0 & 0 & \nu
\end{array}\right),
$$

where $\mathcal{H}$ is spanned by $\xi$ and $X$ at each point.

THEOREM A ([3]). Let $\alpha(t), h(t), \lambda(t), v(t)$ be analytic functions on an open interval

Received March 26, 2015; revised May 24, 2016

Mathematics Subject Classification: 53C40, 53C55, 53C25

Key words and phrases: non-Hopf hypersurface, shape operator, Ricci tensor, complex space form 
$I \subset \mathbf{R}$ satisfying the underdetermined ODE system

$$
\begin{aligned}
\alpha^{\prime} & =h(\alpha+\lambda-3 v), \\
h^{\prime} & =h^{2}+\lambda^{2}-2 \lambda v+\alpha v+c, \\
\lambda^{\prime} & =\frac{(2 \lambda+v) h^{2}+(v-\lambda)\left(\alpha \lambda-\lambda^{2}+c\right)}{h},
\end{aligned}
$$

with $h(t)$ nowhere zero. Let $\gamma(t)$ be a unit-speed analytic framed curve in $M^{2}(c)$, defined for $t \in I$, with transverse curvature $v(t)$, zero holomorphic curvature and zero torsion. Then there exists a non-Hopf hypersurface $M^{3}$ such that

(i) the distribution $\mathcal{H}$ is rank 2 and integrable;

(ii) $M$ has a globally defined frame $\{\xi, X, \phi X\}$ with respect to which the shape operator has the form (1), such that $\alpha, h, \lambda$ and $v$ are constant along the leaves of $\mathcal{H}$, and

(iii) $M$ contains $\gamma$ as a principal curve to which the vector field $Y=\phi X$ is tangent, and along which the restricted components of A coincide with the given solution of the ODE system.

In section 3, we consider a condition on the shape operator that contains the totally $\eta$ umbilical condition. We show that some non-Hopf hypersurfaces related to Theorem A also satisfy this condition. We shall prove

TheOREM 1. Let $M$ be a real hypersurface in $M^{2}(c), c \neq 0$. Suppose there exists a smooth function $a: M \rightarrow \mathbf{R}$ such that $g(A X, Y)=a g(X, Y)$ for any vector fields $X$ and $Y$ orthogonal to the structure vector field $\xi$. Then $M$ is locally congruent to one of the following;

(a) a totally $\eta$-umbilical real hypersurface,

(b) a ruled real hypersurface,

(c) a real hypersurface with the shape operator

$$
A=\left(\begin{array}{lll}
\alpha & h & 0 \\
h & a & 0 \\
0 & 0 & a
\end{array}\right)
$$

with respect to an orthonormal frame $\left\{\xi, e_{1}, \phi e_{1}\right\}$, and for a principal curve $\gamma(t)$ $\left(t \in I, \gamma^{\prime}=\phi e_{1}\right)$, satisfying

$$
\begin{aligned}
& \alpha^{\prime}=h \alpha-2 h a, \\
& h^{\prime}=c-a^{2}+a \alpha+h^{2}, \\
& a^{\prime}=3 h a .
\end{aligned}
$$

The corresponding result for a real hypersurface of $M^{n}(c), n \geq 3, c \neq 0$, is given by Ortega [11]. 
THEOREM B ([11]). Let $M$ be a real hypersurface of $M^{n}(c), n \geq 3, c \neq 0$. Suppose there exists a smooth function $a: M \rightarrow \mathbf{R}$ such that $g(A X, Y)=a g(X, Y)$ for any vector fields $X$ and $Y$ orthogonal to $\xi$. Then $M$ is locally congruent to one of the following:

(a) a totally $\eta$-umbilical real hypersurface,

(b) a ruled real hypersurface.

If the curvature tensor $R$ and the Ricci operator $S$ satisfy $R(X, Y) \cdot S=0$ for any vector fields $X$ and $Y$, then $M$ is called a pseudo-Ryan hypersurface. In [3], as a result of Theorem A, Ivey and Ryan gave an example of a pseudo-Ryan hypersurface in $M^{2}(c)$.

THEOREM C ([3]). Let $\alpha(t), h(t), \lambda(t), v(t)$ be analytic solutions defined on I of the system (2), such that $h$ is nowhere zero and the equation

$$
h^{2} v^{2}+(4 c+\lambda \nu)\left(\alpha(\lambda-v)-h^{2}\right)=0
$$

holds. Then the hypersurface M constructed by Theorem A is a non-Hopf pseudo-Ryan hypersurface.

In Section 4, we consider a condition that the Ricci operator $S$ is pseudo-parallel, that is,

$$
R(X, Y) \cdot S=F(X \wedge Y) \cdot S,
$$

where $F$ is a function, which contains the pseudo-Ryan condition. We define the wedge product $X \wedge Y$ by

$$
(X \wedge Y) Z=g(Y, Z) X-g(X, Z) Y
$$

for vectors $X$ and $Y$. It is shown that a ruled real hypersurface in non-flat complex space form $M^{2}(c), c \neq 0$ cannot have a pseudo-parallel Ricci operator ([2], [6]). In [6], Inoguchi gave a conjecture that real hypersurfaces in a non-flat complex space form $M^{2}(c)$ with pseudoparallel Ricci operator are Hopf. We prove the following theorem which gives the negative result.

THEOREM 2. Let $M$ be a real hypersurface in $M^{2}(c), c \neq 0$. If the Ricci operator $S$ is pseudo-parallel, then $M$ is a Hopf hypersurface or a non-Hopf hypersurface such that the shape operator has the form

$$
A=\left(\begin{array}{ccc}
\alpha & h & 0 \\
h & a_{1} & 0 \\
0 & 0 & a_{2}
\end{array}\right)
$$

with respect to an orthonormal frame $\left\{\xi, e_{1}, e_{2}\right\}$, and

$$
0=\left(a_{2} \alpha-a_{1} \alpha+h^{2}\right)\left(3 c+a_{1} a_{2}-a_{1} \alpha+h^{2}\right)-a_{2}^{2} h^{2},
$$




$$
F=c+a_{1} \alpha-h^{2} .
$$

If there exists a function $F$ such that

$$
g((R(X, Y) S) Z, W)=F g(((X \wedge Y) S) Z, W),
$$

for all $X, Y, Z$ and $W$ orthogonal to $\xi$, then the real hypersurface is said to be pseudo $\eta$ parallel, which is a weaker condition than pseudo-parallel. When $M$ is a real hypersurface of $M^{n}(c), n \geq 3, c \neq 0$, the author showed the following.

THEOREM D ([7]). Let $M$ be a real hypersurface in a complex space form $M^{n}(c)$, $c \neq 0, n \geq 3$. Then the Ricci operator $S$ is pseudo $\eta$-parallel if and only if $M$ is pseudoEinstein.

We remark that a pseudo-Einstein real hypersurface is a Hopf hypersurface.

\section{Preliminaries}

Let $M^{n}(c)$ denote the complex space form of complex dimension $n$ (real dimension $2 n$ ) with constant holomorphic sectional curvature $4 c$. We denote by $J$ the almost complex structure of $M^{n}(c)$. The Hermitian metric of $M^{n}(c)$ will be denoted by $G$.

Let $M$ be a real $(2 n-1)$-dimensional hypersurface immersed in $M^{n}(c)$. We denote by $g$ the Riemannian metric induced on $M$ from $G$. We take the unit normal vector field $N$ of $M$ in $M^{n}(c)$. For any vector field $X$ tangent to $M$, we define $\phi, \eta$ and $\xi$ by

$$
J X=\phi X+\eta(X) N, \quad J N=-\xi,
$$

where $\phi X$ is the tangential part of $J X, \phi$ is a tensor field of type $(1,1), \eta$ is a 1 -form, and $\xi$ is the unit vector field on $M$. Then they satisfy

$$
\phi^{2} X=-X+\eta(X) \xi, \quad \phi \xi=0, \quad \eta(\phi X)=0
$$

for any vector field $X$ tangent to $M$. Moreover, we have

$$
\begin{aligned}
& g(\phi X, Y)+g(X, \phi Y)=0, \quad \eta(X)=g(X, \xi), \\
& g(\phi X, \phi Y)=g(X, Y)-\eta(X) \eta(Y) .
\end{aligned}
$$

Thus $(\phi, \xi, \eta, g)$ defines an almost contact metric structure on $M$.

We denote by $\tilde{\nabla}$ the operator of covariant differentiation in $M^{n}(c)$, and by $\nabla$ the one in $M$ determined by the induced metric. Then the Gauss and Weingarten formulas are given respectively by

$$
\tilde{\nabla}_{X} Y=\nabla_{X} Y+g(A X, Y) N, \quad \tilde{\nabla}_{X} N=-A X
$$

for any vector fields $X$ and $Y$ tangent to $M$. We call $A$ the shape operator of $M$. If the shape operator $A$ of $M$ satisfies $A \xi=\alpha \xi$ for some functions $\alpha$, then $M$ is called a Hopf hypersurface. 
For the almost contact metric structure on $M$, we have

$$
\nabla_{X} \xi=\phi A X, \quad\left(\nabla_{X} \phi\right) Y=\eta(Y) A X-g(A X, Y) \xi .
$$

If the shape operator $A$ of a real hypersurface $M$ is of the form $A=a I$, where $I$ is the identity, then $M$ is said to be totally umbilical. In Tashiro-Tachibana [13], it was proved that any real hypersurface of $M^{n}(c), c \neq 0$, is not totally umbilical. So we need the notion of totally $\eta$ umbilical real hypersurfaces, that is, the shape operator $A$ is of the form $A=a I+b \eta \otimes \xi$.

PROPOSITION E ([12]). The only totally $\eta$-umbilical real hypersurfaces in $\mathbf{C} P^{n}, n \geq$ 2, are geodesic hyperspheres.

Proposition F ([9], [10]). The only totally $\eta$-umbilical real hypersurfaces in $\mathbf{C} H^{n}$, $n \geq 2$, are horospheres, geodesic hyperspheres and tubes over complex hyperbolic hyperplane.

We denote by $R$ the Riemannian curvature tensor field of $M$. Then the equation of Gauss is given by

$$
\begin{aligned}
R(X, Y) Z= & c\{g(Y, Z) X-g(X, Z) Y+g(\phi Y, Z) \phi X \\
& -g(\phi X, Z) \phi Y-2 g(\phi X, Y) \phi Z\} \\
& +g(A Y, Z) A X-g(A X, Z) A Y,
\end{aligned}
$$

and the equation of Codazzi by

$$
\left(\nabla_{X} A\right) Y-\left(\nabla_{Y} A\right) X=c\{\eta(X) \phi Y-\eta(Y) \phi X-2 g(\phi X, Y) \xi\} .
$$

From the equation of Gauss, the Ricci operator $S$ of $M$ satisfies

$$
\begin{aligned}
g(S X, Y)= & (2 n+1) c g(X, Y)-3 c \eta(X) \eta(Y) \\
& +\operatorname{Tr} A g(A X, Y)-g(A X, A Y),
\end{aligned}
$$

where $\operatorname{Tr} A$ is the trace of $A$. The scalar curvature $r$ is defined by

$$
r=\operatorname{Tr} S \text {. }
$$

EXAMPLE ([5], [8]). Let $M$ be a real hypersurface of a complex space form $M^{n}(c)$, $c \neq 0$, and let $T_{0}$ be the distribution defined by $T_{0}(x)=\left\{X \in T_{x}(M) \mid X \perp \xi\right\}$ for $x \in M$. If $T_{0}$ is integrable and its integral manifold is a totally geodesic submanifold $M^{n-1}(c)$, then $M$ is called a ruled real hypersurface. Let $\gamma(t)(t \in I)$ be an arbitrary (regular) curve in $M^{n}(c)$. Then for every $t \in I$ there exists a totally geodesic submanifold $M^{n-1}(c)$ in $M^{n}(c)$ which is orthogonal to the plane $\tau_{t}$ spanned by $\left\{\gamma^{\prime}(t), J \gamma^{\prime}(t)\right\}$. Here we denote by $M_{t}^{n-1}(c)$ such a totally geodesic submanifold. Let $M=\left\{x \in M_{t}^{n-1}(c) \mid t \in I\right\}$. Then the construction of $M$ asserts that $M$ is a ruled real hypersurface in $M^{n}(c)$. Moreover, the construction of $M$ tells 
that there are many ruled real hypersurfaces. The holomorphic sectional curvature $H$ of a ruled real hypersurface $M$ is $4 c$ (see [4]).

\section{A condition of shape operator}

In this section, we prove Theorem 1. As a consequence of this theorem, we have the following.

COROLlary 1. Let $M$ be a real hypersurface in $M^{2}(c), c \neq 0$. Suppose there exists a constant $a: M \rightarrow \mathbf{R}$ such that $g(A X, Y)=a g(X, Y)$ for any vector fields $X$ and $Y$ orthogonal to $\xi$. Then $M$ is locally congruent to one of the following:

(a) a totally $\eta$-umbilical real hypersurface,

(b) a ruled real hypersurface.

First we prove the following

LEMMA 1. Let $M$ be a real hypersurface in $M^{2}(c)$. Suppose that there exists a smooth function $a: M \rightarrow \mathbf{R}$ such that $g(A X, Y)=a g(X, Y)$ for any vector fields $X$ and $Y$ orthogonal to $\xi$, then $M$ is a Hopf hypersurface or the shape operator $A$ is represented by a matrix

$$
A=\left(\begin{array}{lll}
\alpha & h & 0 \\
h & a & 0 \\
0 & 0 & a
\end{array}\right)
$$

with respect to a suitable orthonormal frame $\{\xi, u, \phi u\}$, locally.

PROOF. By the assumption, we can take an orthonormal frame $\left\{\xi, e_{1}, e_{2}=\phi e_{1}\right\}$, such that $A$ is represented by a matrix

$$
A=\left(\begin{array}{ccc}
\alpha & k_{1} & k_{2} \\
k_{1} & a & 0 \\
k_{2} & 0 & a
\end{array}\right),
$$

locally, for suitable functions $k_{1}, k_{2}$ and $\alpha$. We take a unit vector $u$ that satisfies

$$
A \xi=\alpha \xi+h u, \quad g(\xi, u)=0,
$$

where $h$ is a function. Then $\{\xi, u, \phi u\}$ is another orthonormal frame of $T_{x}(M)$. We can represent $u$ as

$$
u=u_{1} e_{1}+u_{2} e_{2} .
$$

Using this, we have

$$
\begin{aligned}
g(A u, u) & =g\left(A\left(u_{1} e_{1}+u_{2} e_{2}\right), u_{1} e_{1}+u_{2} e_{2}\right) \\
& =u_{1}^{2} g\left(A e_{1}, e_{1}\right)+2 u_{1} u_{2} g\left(A e_{1}, e_{2}\right)+u_{2}^{2} g\left(A e_{2}, e_{2}\right)
\end{aligned}
$$




$$
=a\left(u_{1}^{2}+u_{2}^{2}\right)=a .
$$

Similarly, we also have $g(A \phi u, \phi u)=a$. Moreover, we obtain

$$
\begin{aligned}
g(A u, \phi u) & =g\left(A\left(u_{1} e_{1}+u_{2} e_{2}\right), u_{1} \phi e_{1}+u_{2} \phi e_{2}\right) \\
& =g\left(A\left(u_{1} e_{1}+u_{2} e_{2}\right), u_{1} e_{2}-u_{2} e_{1}\right) \\
& =-u_{1} u_{2} a+u_{2} u_{1} a=0 .
\end{aligned}
$$

From these equations, there exists an orthonormal frame $\{\xi, u, \phi u\}$ of $T_{x}(M)$ such that the shape operator $A$ is of the form (5).

Using the equation of Codazzi, we obtain

LEMMA 2. Let $M$ be a real hypersurface in $M^{2}(c), c \neq 0$. If there exists an orthonormal frame $\left\{\xi, e_{1}, e_{2}\right\}$ on a sufficiently small neighborhood $\mathcal{N}$ of $x \in M$ such that the shape operator $A$ can be represented as

$$
A=\left(\begin{array}{lll}
\alpha & h & 0 \\
h & a & 0 \\
0 & 0 & a
\end{array}\right),
$$

then we have

$$
\begin{aligned}
& \left(e_{1} a\right)=0 \\
& \left(-2 c+2 a^{2}-2 a \alpha\right)+h g\left(\nabla_{e_{1}} e_{2}, e_{1}\right)+\left(e_{2} h\right)=0 \\
& \left(e_{2} a\right)=3 h a \\
& (\xi a)=h g\left(\nabla_{e_{2}} e_{1}, e_{2}\right) \\
& \left(e_{2} h\right)=c+a \alpha-a^{2}+h^{2} \\
& -h(\alpha-3 a)+h g\left(\nabla_{\xi} e_{2}, e_{1}\right)+\left(e_{2} \alpha\right)=0 \\
& \left(e_{1} h\right)-(\xi a)=0 \\
& \left(e_{1} \alpha\right)-(\xi h)=0
\end{aligned}
$$

Proof. By the equation of Codazzi, we have

$$
g\left(\left(\nabla_{e_{2}} A\right) e_{1}-\left(\nabla_{e_{1}} A\right) e_{2}, e_{2}\right)=0 .
$$

On the other hand, we have

$$
\begin{aligned}
& g\left(\left(\nabla_{e_{2}} A\right) e_{1}-\left(\nabla_{e_{1}} A\right) e_{2}, e_{2}\right) \\
& \quad=g\left(\nabla_{e_{2}}\left(A e_{1}\right)-A \nabla_{e_{2}} e_{1}-\nabla_{e_{1}}\left(A e_{2}\right)+A \nabla_{e_{1}} e_{2}, e_{2}\right) \\
& \quad=-\left(e_{1} a\right) .
\end{aligned}
$$

Thus we obtain (6). By the similar computation, we have our equations. 
When $M$ is not a Hopf hypersurface, then we can take $x \in M$ and a sufficiently small neighborhood of $x$, on which $h \neq 0$. In the following, we consider the case that $a \neq 0$ on the neighborhood.

LEMMA 3. If $h \neq 0$ and $a \neq 0$, then,

$$
\begin{aligned}
& \nabla_{e_{1}} e_{1}=\frac{-c+a^{2}-a \alpha+h^{2}}{h} e_{2}, \\
& \nabla_{e_{1}} e_{2}=\frac{c-a^{2}+a \alpha-h^{2}}{h} e_{1}-a \xi, \\
& \nabla_{e_{2}} e_{1}=a \xi, \quad \nabla_{e_{2}} e_{2}=0, \\
& \nabla_{\xi} e_{1}=a e_{2}, \quad \nabla_{\xi} e_{2}=-a e_{1}-h \xi .
\end{aligned}
$$

Moreover, we have

$$
\begin{aligned}
& e_{1} a=0, \quad e_{1} h=0, \quad e_{1} \alpha=0, \\
& e_{2} a=3 h a, \quad e_{2} h=c-a^{2}+a \alpha+h^{2}, \quad e_{2} \alpha=h \alpha-2 h a, \\
& \xi a=0, \quad \xi h=0, \quad \xi \alpha=0 .
\end{aligned}
$$

PROOF. First we compute $\nabla_{e_{1}} e_{2}$. Using (7) and (10), we have

$$
g\left(\nabla_{e_{1}} e_{2}, e_{1}\right)=-g\left(\nabla_{e_{1}} e_{1}, e_{2}\right)=\frac{c-a^{2}+a \alpha-h^{2}}{h} .
$$

Moreover, we obtain $g\left(\nabla_{e_{1}} e_{2}, e_{2}\right)=0$ and

$$
g\left(\nabla_{e_{1}} e_{2}, \xi\right)=-g\left(e_{2}, \phi A e_{1}\right)=-a .
$$

So we have

$$
\nabla_{e_{1}} e_{2}=\frac{c-a^{2}+a \alpha-h^{2}}{h} e_{1}-a \xi .
$$

By the similar computation using Lemma 2, we obtain

$$
\begin{aligned}
& \nabla_{e_{2}} e_{1}=\frac{(\xi a)}{h} e_{2}+a \xi, \\
& \nabla_{e_{1}} e_{1}=\frac{-c+a^{2}-a \alpha+h^{2}}{h} e_{2}, \\
& \nabla_{e_{2}} e_{2}=-\frac{(\xi a)}{h} e_{1} .
\end{aligned}
$$

We put $g\left(\nabla_{\xi} e_{1}, e_{2}\right)=P$. Then we have

$$
\begin{aligned}
& \nabla_{\xi} e_{1}=P e_{2}, \\
& \nabla_{\xi} e_{2}=-P e_{1}-h \xi .
\end{aligned}
$$


Since $[X, Y]=\nabla_{X} Y-\nabla_{Y} X$ for any $X$ and $Y$ tangent to $M$, we have

$$
\begin{aligned}
{\left[e_{1}, e_{2}\right] a } & =\left(\nabla_{e_{1}} e_{2}-\nabla_{e_{2}} e_{1}\right) a \\
& =\frac{c-a^{2}+a \alpha-h^{2}}{h}\left(e_{1} a\right)-a(\xi a)-\frac{(\xi a)}{h}\left(e_{2} a\right)-a(\xi a) \\
& =-5 a(\xi a) .
\end{aligned}
$$

For the last equality, we use (6) and (8). On the other hand, by (6) and (12), we obtain

$$
\begin{aligned}
{\left[e_{1}, e_{2}\right] a } & =e_{1}\left(e_{2} a\right)-e_{2}\left(e_{1} a\right)=e_{1}(3 h a) \\
& =3\left(e_{1} h\right) a+3 h\left(e_{1} a\right)=3(\xi a) a .
\end{aligned}
$$

These equations imply $a(\xi a)=0$, and hence

$$
(\xi a)=\left(e_{1} h\right)=0 .
$$

Similarly, we have

$$
\begin{aligned}
{\left[e_{1}, \xi\right] a } & =\left(\nabla_{e_{1}} \xi-\nabla_{\xi} e_{1}\right) a \\
& =3 h a(a-P) .
\end{aligned}
$$

Using (6) and (14), we obtain

$$
\left[e_{1}, \xi\right] a=e_{1}(\xi a)-\xi\left(e_{1} a\right)=0 .
$$

Since $h a \neq 0$, we have $a=P$. Thus, by (11),

$$
\left(e_{2} \alpha\right)=h \alpha-2 h a \text {. }
$$

By the similar computation for $\left[e_{2}, \xi\right] a$ and $\left[e_{2}, \xi\right] h$, we also have

$$
(\xi h)=0, \quad\left(e_{1} \alpha\right)=0, \quad(\xi \alpha)=0 .
$$

Combining these results, we have our assertion.

(Proof of Theorem 1)

When $M$ is a Hopf hypersurface, then we have $A X=a X+b \eta(X) \xi$ for some function $b$. This means that $M$ is totally $\eta$-umbilical.

Next we consider the case that $M$ is not Hopf. Then we can take a point $x$ and a sufficiently small neighborhood of $x$, on which $h \neq 0$. If $a=0$ on the neighborhood, we see that the real hypersurface is locally congruent to a ruled real hypersurface.

Finally, we suppose $h a \neq 0$. We can take a unit-speed analytic framed curve $\gamma(t)$ which satisfy $\gamma^{\prime}=e_{2}$. Then Lemma 3 shows that $a, h$ and $\alpha$ satisfy (3). We note that the existence of this non-Hopf hypersurface is induced by Theorem A.

Conversely, such hypersurfaces satisfy the condition $g(A X, Y)=a g(X, Y)$ for any vector fields $X$ and $Y$ orthogonal to $\xi$. 


\section{3-dimensional real hypersurfaces with pseudo-parallel Ricci operator}

If the Ricci operator $S$ of a real hypersurface $M$ satisfies

$$
R(X, Y) \cdot S=F(X \wedge Y) \cdot S,
$$

where $F$ is a function, then the Ricci operator $S$ is said to be pseudo-parallel.

To prove Theorem 2, first we show the following.

LEMMA 4. Let $M$ be a real hypersurface in $M^{2}(c), c \neq 0$. If the Ricci operator $S$ is pseudo-parallel, then $M$ is a Hopf hypersurface or the shape operator A is represented by the matrix

$$
A=\left(\begin{array}{ccc}
\alpha & h & 0 \\
h & a_{1} & 0 \\
0 & 0 & a_{2}
\end{array}\right)
$$

with respect to an orthonormal frame $\left\{\xi, e_{1}, e_{2}\right\}$, locally.

Proof. Suppose that $M$ is not a Hopf hypersurface. We take an orthonormal frame $\left\{\xi, e_{1}, e_{2}\right\}$, where we have put $e_{2}=\phi e_{1}$. Then there are smooth functions $a_{1}, a_{2}, h_{1}$ and $h_{2}$ such that $A$ is represented by a matrix

$$
A=\left(\begin{array}{ccc}
\alpha & h_{1} & h_{2} \\
h_{1} & a_{1} & 0 \\
h_{2} & 0 & a_{2}
\end{array}\right)
$$

with respect to $\left\{\xi, e_{1}, e_{2}\right\}$, locally. We remark that $h_{1} \neq 0$ or $h_{2} \neq 0$. From (4), we have

$$
\begin{aligned}
& S e_{1}=\left(5 c+a_{1} a_{2}+a_{1} \alpha-h_{1}^{2}\right) e_{1}-h_{1} h_{2} e_{2}+a_{2} h_{1} \xi, \\
& S e_{2}=\left(5 c+a_{1} a_{2}+a_{2} \alpha-h_{2}^{2}\right) e_{2}-h_{1} h_{2} e_{1}+a_{1} h_{2} \xi, \\
& S \xi=a_{2} h_{1} e_{1}+a_{1} h_{2} e_{2}+\left(2 c+a_{1} \alpha+a_{2} \alpha-h_{1}^{2}-h_{2}^{2}\right) \xi .
\end{aligned}
$$

Since $S$ is symmetric, there exists an another orthonormal frame $\left\{v_{1}, v_{2}, v_{3}\right\}$ that satisfies $S v_{1}=a v_{1}, S v_{2}=b v_{2}, S v_{3}=d v_{3}$ for some functions $a, b$ and $d$. Since $S$ is pseudo-parallel, we have

$$
\begin{aligned}
& g(R(X, Y) S Z, W)-g(S R(X, Y) Z, W) \\
&= F\{g(Y, S Z)(X, W)-g(X, S Z) g(Y, W)-g(Y, Z) g(S X, W) \\
&+g(X, Z) g(S Y, W)\} .
\end{aligned}
$$

Putting $X=W=v_{1}$ and $Y=Z=v_{2}$, we obtain

$$
(b-a)\left(K\left(v_{1}, v_{2}\right)-F\right)=0,
$$

where the sectional curvature $K$ for the plane spanned by $v_{1}$ and $v_{2}$ is denoted by

$$
K\left(v_{1}, v_{2}\right)=g\left(R\left(v_{1}, v_{2}\right) v_{2}, v_{1}\right) .
$$


By the similar computation, we have

$$
\begin{aligned}
& (d-a)\left(K\left(v_{1}, v_{3}\right)-F\right)=0, \\
& (d-b)\left(K\left(v_{2}, v_{3}\right)-F\right)=0 .
\end{aligned}
$$

If $a \neq b, b \neq c$ and $c \neq a$, then we see that

$$
F=K\left(v_{1}, v_{2}\right)=K\left(v_{1}, v_{3}\right)=K\left(v_{2}, v_{3}\right) .
$$

Thus we obtain

$$
\begin{aligned}
a & =g\left(S e_{1}, e_{1}\right)=K\left(v_{1}, v_{2}\right)+K\left(v_{1}, v_{3}\right) \\
& =K\left(v_{1}, v_{2}\right)+K\left(v_{2}, v_{3}\right) \\
& =g\left(S e_{2}, e_{2}\right)=b .
\end{aligned}
$$

This is a contradiction. From the fact that no real hypersurfaces of $M^{2}(c)$ are Einstein, it is sufficient to consider the case that $a=b \neq d$. Then we have

$$
F=K\left(v_{1}, v_{3}\right)=K\left(v_{2}, v_{3}\right)
$$

from which

$$
g\left(S v_{3}, v_{3}\right)=K\left(v_{1}, v_{3}\right)+K\left(v_{2}, v_{3}\right)=d=2 F .
$$

So the Ricci operator $S$ is represented by a matrix

$$
S=\left(\begin{array}{ccc}
a & 0 & 0 \\
0 & a & 0 \\
0 & 0 & 2 F
\end{array}\right)
$$

with respect to $\left\{v_{1}, v_{2}, v_{3}\right\}$.

On the other hand, from the assumption, we have

$$
g\left(\left(R\left(e_{1}, e_{2}\right) S\right) e_{1}, e_{1}\right)=F g\left(\left(\left(e_{1} \wedge e_{2}\right) \cdot S\right) e_{1}, e_{1}\right) .
$$

By the equation of Gauss and (15), we obtain

$$
\begin{aligned}
g\left(\left(R\left(e_{1}, e_{2}\right) S\right) e_{1}, e_{1}\right)= & g\left(R\left(e_{1}, e_{2}\right) S e_{1}, e_{1}\right)-g\left(R\left(e_{1}, e_{2}\right) e_{1}, S e_{1}\right) \\
= & 2 c\left(g\left(e_{2}, S e_{1}\right) g\left(e_{1}, e_{1}\right)-g\left(\phi e_{1}, S e_{1}\right)\left(\phi e_{2}, e_{1}\right)\right. \\
& \left.-2 g\left(\phi e_{1}, e_{2}\right) g\left(\phi S e_{1}, e_{1}\right)\right) \\
& +2 g\left(A e_{2}, S e_{1}\right) g\left(A e_{1}, e_{1}\right) \\
= & -8 c h_{1} h_{2} .
\end{aligned}
$$

By (15), we have

$$
\begin{aligned}
F g\left(\left(\left(e_{1} \wedge e_{2}\right) \cdot S\right) e_{1}, e_{1}\right)= & F\left(g\left(e_{2}, S e_{1}\right)\left(e_{1}, e_{1}\right)-g\left(S e_{1}, e_{1}\right) g\left(e_{2}, e_{1}\right)-g\left(e_{2}, e_{1}\right) g\left(S e_{1}, e_{1}\right)\right. \\
& \left.+g\left(e_{1}, e_{1}\right) g\left(S e_{2}, e_{1}\right)\right)
\end{aligned}
$$




$$
=2 F\left(S e_{1}, e_{2}\right)=-2 F h_{1} h_{2} .
$$

From these equations, we see that

$$
(4 c-F) h_{1} h_{2}=0 .
$$

Similarly, substituting $X=Z=e_{1}, Y=\xi, W=e_{2}$ and $X=Z=e_{2}, Y=\xi, W=e_{1}$, we obtain

$$
\begin{aligned}
& 0=h_{2}\left\{(c-F) a_{1}-a_{2} h_{1}^{2}+a_{1} a_{2} \alpha-a_{1} h_{2}^{2}\right\}, \\
& 0=h_{1}\left\{(c-F) a_{2}-a_{1} h_{2}^{2}+a_{1} a_{2} \alpha-a_{2} h_{1}^{2}\right\},
\end{aligned}
$$

respectively.

To prove the lemma, it is sufficient to consider the case that $h_{1} h_{2} \neq 0$. From (18) and (19), we have $4 c=F$ and

$$
(c-F)\left(a_{1}-a_{2}\right)=0 .
$$

Since $c-F=-3 c \neq 0$, we obtain $a_{1}=a_{2}$. By Lemma 1 , the shape operator $A$ is represented as

$$
A=\left(\begin{array}{lll}
\alpha & h & 0 \\
h & k & 0 \\
0 & 0 & k
\end{array}\right)
$$

with respect to an orthonormal frame $\{\xi, u, \phi u\}$. Thus we have our assertion.

(Proof of Theorem 2)

Suppose that $M$ is not a Hopf hypersurface. We put $h_{1}=h \neq 0$, locally. Then the Ricci operator $S$ is represented by a matrix

$$
S=\left(\begin{array}{ccc}
2 c+a_{1} \alpha+a_{2} \alpha-h^{2} & a_{2} h & 0 \\
a_{2} h & 5 c+a_{1} a_{2}+a_{1} \alpha-h^{2} & 0 \\
0 & 0 & 5 c+a_{1} a_{2}+a_{2} \alpha
\end{array}\right)
$$

with respect to $\left\{\xi, e_{1}, e_{2}\right\}$. By (17), we see that $5 c+a_{1} a_{2}+a_{2} \alpha=2 F$ or $5 c+a_{1} a_{2}+a_{2} \alpha=a$.

First we suppose $5 c+a_{1} a_{2}+a_{2} \alpha=2 F$. From (17) and (21), taking a trace of $S$, the scalar curvature $r$ satisfies

$$
r=2(a+F)=12 c+2 a_{1} a_{2}+2 a_{1} \alpha+2 a_{2} \alpha-2 h^{2} .
$$

So we have

$$
a=F+c+a_{1} \alpha-h^{2} .
$$

We put

$$
S^{\prime}=\left(\begin{array}{cc}
2 c+a_{1} \alpha+a_{2} \alpha-h^{2} & a_{2} h \\
a_{2} h & 5 c+a_{1} a_{2}+a_{1} \alpha-h^{2}
\end{array}\right) .
$$


Then the eigenvalues of $S^{\prime}$ are solutions of the equation

$$
\begin{aligned}
0= & \operatorname{det}\left(x I-S^{\prime}\right) \\
= & \left(x-5 c-a_{1} a_{2}-a_{1} \alpha+h^{2}\right)\left(x-2 c-a_{1} \alpha-a_{2} \alpha+h^{2}\right) \\
& -a_{2}^{2} h^{2} .
\end{aligned}
$$

Since $a$ is an eigenvalue of $S^{\prime}$, using (22), we have

$$
0=\left(F-4 c-a_{1} a_{2}\right)\left(F-c-a_{2} \alpha\right)-a_{2}^{2} h^{2} .
$$

By $a_{1} a_{2}=2 F-5 c-a_{2} \alpha$, we obtain

$$
0=-\left(F-c-a_{2} \alpha\right)^{2}-a_{2}^{2} h^{2},
$$

which induces $F-c-a_{2} \alpha=0$ and $a_{2}^{2} h^{2}=0$. Since $h \neq 0$, we have $a_{2}=0$ and $F=c$. By $5 c+a_{1} a_{2}+a_{2} \alpha=2 F$, we have

$$
0=2 F-5 c=-3 c .
$$

This is a contradiction.

Next, we suppose $5 c+a_{1} a_{2}+a_{2} \alpha=a$. Then we have

$$
r=2(a+F)=2\left(6 c+a_{1} a_{2}+a_{1} \alpha+a_{2} \alpha-h^{2}\right) .
$$

From these equations, we have

$$
F=c+a_{1} \alpha-h^{2} .
$$

Since $a$ and $2 F$ are the solutions of (23), we obtain

$$
0=\left(a_{2} \alpha-a_{1} \alpha+h^{2}\right)\left(3 c+a_{1} a_{2}-a_{1} \alpha+h^{2}\right)-a_{2}^{2} h^{2} .
$$

So we see that if $S$ is pseudo-parallel, then $M$ is a Hopf hypersurface or the shape operator $A$ is represented by

$$
A=\left(\begin{array}{ccc}
\alpha & h & 0 \\
h & a_{1} & 0 \\
0 & 0 & a_{2}
\end{array}\right)
$$

with respect to an orthonormal frame $\left\{\xi, e_{1}, e_{2}\right\}$ and satisfies (24), (25). So we have our theorem.

In [2], Cho, Hamada and Inoguchi gave a classification of pseudo-parallel Hopf hypersurfaces.

TheOREM G ([2]). The Hopf hypersurfaces in $\mathbf{C} P^{2}(c)$ or $\mathbf{C} H^{2}(c)$ with pseudoparallel Ricci operator are locally holomorphically congruent to a horosphere in $\mathbf{C} H^{2}(c)$, a geodesic hypersphere in $\mathbf{C} P^{2}(c)$ or $\mathbf{C} H^{2}(c)$, a homogeneous tube over $\mathbf{C H} H^{1}(c)$ in $\mathbf{C} H^{2}, a$ 
non-homogeneous real hypersurface which is realized as a tube over a certain holomorphic curve in $\mathbf{C} P^{2}(c)$ with radius $\pi / \sqrt{4 c}$, or a Hopf hypersurface in $\mathbf{C} H^{2}(c)$ with $A \xi=0$.

Using Theorem A, we see the following result (see Corollary 3 in [3]).

COROLlaRy 2. Let $\alpha(t), h(t), \lambda(t), v(t)$ be analytic solutions defined for $t \in I$ of the system (2), such that $h$ is nowhere zero and

$$
0=\left(v \alpha-\lambda \alpha+h^{2}\right)\left(3 c+\lambda v-\lambda \alpha+h^{2}\right)-v^{2} h^{2} .
$$

Then the hypersurface $M$ constructed by Theorem A is a non-Hopf pseudo-parallel hypersurface with $F=c+\lambda \alpha-h^{2}$.

Proof. We suppose that $A$ satisfies (24)-(26) and $a_{1}=\lambda, a_{2}=v$. It is sufficient to show that

$$
g((R(X, Y) S) Z, W)-F g(((X \wedge Y) S) Z, W)=0
$$

for all $X=e_{i}, Y=e_{j}, Z=e_{k}$ and $W=e_{l}, 1 \leq i, j, k, l \leq 3$, where $e_{3}=\xi$. Using (15), (24)-(26) and the equation of Gauss, we have

$$
\begin{aligned}
& g\left(\left(R\left(e_{1}, e_{2}\right) S\right) e_{1}, e_{2}\right)-F g\left(\left(\left(e_{1} \wedge e_{2}\right) S\right) e_{1}, e_{2}\right) \\
&= g\left(R\left(e_{1}, e_{2}\right) S e_{1}, e_{2}\right)-g\left(R\left(e_{1}, e_{2}\right) e_{1}, S e_{2}\right) \\
&-F\left(-g\left(S e_{1}, e_{1}\right)+g\left(S e_{2}, e_{2}\right)\right) \\
&=-4 c g\left(S e_{1}, e_{1}\right)+g\left(A e_{2}, S e_{1}\right) g\left(A e_{1}, e_{2}\right)-g\left(A e_{1}, S e_{1}\right) g\left(A e_{2}, e_{2}\right) \\
&+4 c g\left(S e_{2}, e_{2}\right)-g\left(A e_{1}, S e_{2}\right) g\left(A e_{2}, e_{1}\right)+g\left(A e_{1}, e_{1}\right) g\left(A e_{2}, S e_{2}\right) \\
&-F\left(-g\left(S e_{1}, e_{1}\right)+g\left(S e_{2}, e_{2}\right)\right) \\
&=\left(a_{2} \alpha-a_{1} \alpha+h^{2}\right)\left(4 c-F+a_{1} a_{2}\right)-a_{2}^{2} h^{2} \\
&=\left(a_{2} \alpha-a_{1} \alpha+h^{2}\right)\left(3 c+a_{1} a_{2}-a_{1} \alpha+h^{2}\right)-a_{2}^{2} h^{2} \\
&= 0 .
\end{aligned}
$$

Similarly, for all $X=e_{i}, Y=e_{j}, Z=e_{k}$ and $W=e_{l}$, we can show that

$$
g((R(X, Y) S) Z, W)-F g(((X \wedge Y) S) Z, W)=0
$$

by the straightforward computation.

REMARK. If the shape operator $A$ satisfies (25), (26) and $a_{1}=a_{2}=0$, then we have $c=h=0$ by (10). Thus a ruled real hypersurface is not pseudo-parallel (see [2] and [6]).

\section{References}

[ 1] J. BERNDT, Real hypersurfaces with constant principal curvatures in complex hyperbolic space, J. Reine Angew. Math. 395 (1989), 132-141.

[ 2 ] J. T. Cho, T. HAmAdA and J. Inoguchi, On three dimensional real hypersurfaces in complex space forms, Tokyo J. Math. 33 (2010), 31-47. 
[ 3 ] T. A. IVEY and P. J. RyAn, The *-Ricci tensor for hypersurfaces in $\mathbf{C P}^{n}$ and $\mathbf{C} H^{n}$, Tokyo J. Math. $\mathbf{3 4}$ (2011), 445-471.

[ 4 ] M. Kimura, Sectional curvatures of holomorphic planes on a real hypersurface in $P^{n}(\mathbf{C})$, Math. Ann. 276 (1987), 487-497.

[ 5 ] M. Kimura and S. MAEdA, On real hypersurfaces of a complex projective space, Math. Z. 202 (1989), 299-311.

[ 6 ] J. INOGUCHI, Real hypersurfaces in complex space forms with pseudo-parallel Ricci operator, Differ. Geom. Dyn. Syst. 14 (2012), 69-89.

[ 7 ] M. Kon, Ricci Pseudo $\eta$-parallel real hypersurfaces of a complex space form, Nihonkai Math. J. 24 (2013), $45-55$.

[ 8 ] M. LohnherR and H. ReCKZIEGEL, On ruled real hypersurfaces in complex space forms, Geom. Dedicata 74 (1999), 267-286.

[ 9 ] S. Montiel and A. Romero, On some real hypersurfaces of a complex hyperbolic space, Geom. Dedicata 20 (1986), 245-261.

[10] R. Niebergall and P. J. RyAn, Real hypersurfaces in complex space forms, Tight and Taut Submanifolds, (Eds: T. E. Cecil and S. S. Chern), Math. Sci. Res. Inst. Publ. 32, Cambridge Univ. Press, Cambridge, 1997, 233-305.

[11] M. ORTEGA, Classifications of real hypersurfaces in complex space forms by means of curvature conditions, Bull. Belg. Math. Soc. 9 (2002), 351-360.

[12] R. TAKAGI, Real hypersurfaces in a complex projective space with constant principal curvatures, J. Math. Soc. Japan 27 (1975), 43-53.

[13] Y. TAShiro and S. TAChibana, On Fubinian and $C$-Fubinian manifolds, Kōdai Math. Sem. Rep. 15 (1963), 176-183.

Present Address:

FACULTY OF EDUCATION,

SHINSHU UNIVERSITY,

6-Ro, Nishinagano, NAGANo City 380-8544, JAPAN.

e-mail: mayuko_k@shinshu-u.ac.jp 